\title{
Prolonged Rupture of Membranes, Neonatal Outcomes and Management Guidelines
}

\author{
Manar Al-lawama ${ }^{\mathrm{a}, \mathrm{b}}$, Ala AlZaatreh ${ }^{\mathrm{a}}$, Rawan Elrajabi ${ }^{\mathrm{a}}$, \\ Sultan Abdelhamid ${ }^{\text {, }}$ Eman Badran ${ }^{\mathrm{a}}$
}

\begin{abstract}
Background: Prolonged rupture of membranes (PROM) is a risk factor for early-onset neonatal sepsis (EOS). In the absence of early specific and sensitive diagnostic tools, management of asymptomatic infants is difficult. This study was conducted to investigate clinical outcomes of newborns born to mothers with PROM.

Methods: A retrospective study of neonates $\geq 34$ weeks admitted due to PROM was conducted. Medical charts were reviewed. Neonates were classified into three categories based on their status at birth: ill appearing, well, and equivocal. Sepsis risk calculator was retrospectively applied.
\end{abstract}

Results: A total of 176 neonates were included. All mothers had unknown group B streptococcus (GBS) status. Of them, 74.4\% were asymptomatic. Nine infants (5\%) had positive cultures, and 23 infants $(13 \%)$ had culture-negative sepsis. The newborns with sepsis fit into the "ill appearing" category with a significantly higher proportion $(12.5 \%$ vs. $0.0 \%$, P value $<0.0)$.

Conclusions: Reliable early diagnostic tools for neonatal sepsis are lacking. Adopting a protocol that utilizes multiple methods and follow-up for the clinical condition of these infants are the key factors to avoid missing neonates with true sepsis and decreasing the use of antibiotics in those without infection.

Keywords: Prolonged rupture of membranes; Sepsis; Neonate; Group B streptococcus; Guidelines

\section{Introduction}

Amniotic membranes are protective to the fetus, with anti-

\footnotetext{
Manuscript submitted March 4, 2019, accepted March 20, 2019

aPediatric Department, School of Medicine, The University of Jordan, Amman, Jordan

${ }^{b}$ Corresponding Author: Manar Al-lawama, Pediatric Department, School of Medicine, The University of Jordan, Amman 11942, Jordan.

Email: manar-76@hotmail.com
}

doi: https://doi.org/10.14740/jocmr3809 inflammatory, anti-bacterial, and anti-viral properties [1]. Prolonged rupture of membranes (PROM) is considered when the duration is more than $18 \mathrm{~h}$ prior to delivery [2]. The incidence of PROM worldwide is variable, ranging from $8 \%$ as recorded by the World Health Organization [3] to around 19\% in countries such as China [4]. The association between neonatal sepsis and the duration of membrane rupture was first reported in 1963 [5] in a study which showed a higher rate of newborns with either clinical or proved sepsis to mothers with ruptured membranes of more than $6 \mathrm{~h}$. Many subsequent studies have linked PROM to neonatal sepsis [6-8]. In contrast, others have dismissed the relation between PROM and neonatal sepsis [9].

In the absence of early specific and sensitive diagnostic tools for neonatal sepsis, management of infants born to mothers with PROM proves to be a dilemma, especially for asymptomatic neonates at birth [10]. Tools such as the Kaiser Permanente early-onset neonatal sepsis (EOS) calculator [11] help physicians calculate the risk of EOS in neonates at 34 weeks' gestational age and older. It has multiple predictors, including the highest maternal temperature reached, maternal group B streptococcus (GBS) status, and antibiotics taken within the intrapartum period. It can be useful in determining which neonates should take antibiotics and which should be under routine care or close observation. However, some are skeptic about the predictability of the calculator, with reports of up to $75 \%$ missed cases of not administering antibiotics to neonates with positive blood cultures when relying on the EOS calculator [12].

The predictability of laboratory investigations such as complete blood count $(\mathrm{CBC})$ and $\mathrm{C}$-reactive protein $(\mathrm{CRP})$ is low especially at the onset of illness, or initially in asymptomatic newborns. Their use to rule out sepsis should depend on serial measurements [13].

Therefore, this diagnostic challenge might justify the practice of admitting all neonates born to mothers with PROM and administering empirical broad-spectrum antibiotics. This is where the problem lies, as many of these asymptomatic neonates do not have sepsis, and yet take empirical antibiotics for an extended period of time. This practice drastically increases the load on neonatal units, and exposes the newborn infants to hospital-acquired infections and medication side effects [14].

In our institution, this issue is further complicated by the lack of antenatal GBS screening as most of our neonates are born to mothers with unknown GBS status. Our current practice is to admit all newborn infants born after PROM to the 
neonatal intensive care unit (NICU) and initiate antibiotics regardless of their symptoms.

The aim of this study is to review the outcome of newborns to mothers with PROM and unknown GBS status and identify significant risk factors that indicate the need for antibiotic administration. We also aim to develop a protocol for management of newborns with PROM.

\section{Materials and Methods}

This is a retrospective study conducted at Jordan University Hospital NICU. The main objective is to evaluate the clinical outcomes of newborns exposed to PROM and their incidence of EOS. In addition, we evaluated the diagnostic utility of the EOS calculator, abnormal laboratory tests, and clinical signs in correctly identifying EOS in neonates born to mothers with PROM.

This study was approved by The University of Jordan Deanship of Scientific Research and by Jordan University Hospital Institutional Review Board (IRB). This study was conducted in compliance with the ethical standards of Jordan University Hospital on human subjects as well as with the Helsinki Declaration.

All admitted neonates born to mothers with PROM between January 2011 and December 2015 were included. They were identified from the hospital data base. Their medical charts were reviewed for demographic and clinical data. Laboratory investigations including blood culture results were also documented. Only neonates who were 34 weeks' gestational age were included in the analysis.

Our unit's protocol is to admit all newborns born to mothers with PROM into the NICU. Blood culture should be obtained at birth and empirical treatment with ampicillin and amikacin should be initiated.

Regarding blood investigations, $\mathrm{CBC}$ is usually done within the first $12 \mathrm{~h}$ in all infants. However, the practice regarding CRP is variable. While it was checked for most infants at $48 \mathrm{~h}$ to guide response to treatment, initial CRP at $6-12 \mathrm{~h}$ of age was not performed for all of them.

Sepsis was defined as the presence of clinical signs and symptoms consistent with sepsis with or without positive blood culture. Culture-negative sepsis was defined as treatment with antibiotics for $\geq 7$ days in neonates with negative blood cultures [15].

Abnormal white blood count was defined as a count above $25 \times 10^{9} / \mathrm{L}$ (leukocytosis) or below $5.0 \times 10^{9} / \mathrm{L}$ (leukopenia) . Thrombocytopenia was defined as a count below $150 \times 10^{9} / \mathrm{L}$, and CRP was considered negative if $<5 \mathrm{mg} / \mathrm{dL}$.

Investigators reviewed the neonates' charts and collected data for retrospective entry into the EOS calculator to determine a base-line risk of EOS at birth. These data included gestational age, duration of maternal membrane rupture, maternal colonization GBS, if the mother received intrapartum antibiotic therapy. If the mother is afebrile, maternal highest temperature was assumed to be $37.0^{\circ} \mathrm{C}$.

Next, investigators classified each neonate as "well, equivocal, or ill" using the clinical signs described on the Kaiser
Table 1. Demographic Characteristics of Neonates $\geq 34$ Weeks' Gestational Age Admitted due to Prolonged Rupture of Membranes

\begin{tabular}{ll}
\hline Character & Distribution \\
\hline Gestational age (mean \pm SD) & $36 \pm 1.7$ \\
\hline Birth weight (mean \pm SD) & $2,767 \pm 777$ \\
Small for age (number/\%) & $9 / 5$ \\
$\begin{array}{l}\text { Male gender (number/\%) } \\
\begin{array}{l}\text { Cesarean section delivery } \\
\text { (number/\%) }\end{array}\end{array}$ & $126 / 71.66$ \\
\hline \multicolumn{1}{|l}{ First min } & $81 / 46.0$ \\
\hline Fifth min & 7 \\
\hline
\end{tabular}

Permanente website; (https://neonatalsepsiscalculator.kaiserpermanente.org). Newborns with symptoms of mild to moderate respiratory distress were classified as equivocal although they were treated with nasal continuous positive airway pressure (CPAP) and should be considered as "ill" according to the above mentioned sepsis calculator. The reason for this is that all newborns with such presentation are managed by nasal CPAP in our unit. Each neonate's EOS risk was determined using the EOS calculator, with the "incidence of EOS" variable set at the highest incidence provided by the calculator $(4 / 1,000$ live births) as neonatal sepsis is still a significant morbidity in Jordan. This way, the generated recommendation will carry the lowest threshold for antibiotic treatment and will guarantee a safer approach. We compared culture-proven and culture-negative septic neonates with those whose sepsis was ruled out in regards to presentation, laboratory results, and the sepsis risk calculator recommendation.

\section{Statistical analysis}

Frequencies were compared using Chi-square test. Comparisons between groups' means were performed using the $t$-test. Statistical significance was set at $\mathrm{P}<0.05$.

\section{Results}

One hundred seventy-six neonates with PROM at 34 weeks' gestational age and above were included. Mean gestational age is $36 \pm 1.7$ weeks. Mean birth weight is $2,767 \pm 777 \mathrm{~g}$. Most of them were of male gender $(71.66 \%)$ (Table 1$)$. Duration of membrane rupture was mostly $<2$ days $(57.3 \%)$, and all mothers had unknown GBS status (Table 2).

Of them, $74.4 \%$ were completely asymptomatic at birth. Nine infants (5\%) had positive cultures, and 23 infants $(13 \%)$ had culture negative sepsis (Table 3 ). The newborns with sepsis fit into the "ill appearing" category with a significantly higher proportion $(12.5 \%$ vs. $0.0 \%$, P value $=0.000)$, and had higher rates of antibiotics prior to delivery (40.6\% vs. $23.6 \%$, 
Table 2. Infection Risk Factors in Neonates $\geq 34$ Weeks' Gestational Age Admitted due to Prolonged Rupture of Membranes

\begin{tabular}{|c|c|}
\hline Characteristic & Number (frequency) \\
\hline \multicolumn{2}{|l|}{ PROM } \\
\hline $\mathrm{PROM}<2$ days & $101(57.3)$ \\
\hline PROM 2 - 6 days & $36(20.5)$ \\
\hline PROM $>7$ days & $39(22.2)$ \\
\hline Maternal UTI & $26(14.8)$ \\
\hline Unknown GBS status & $176(100)$ \\
\hline Maternal chorioamnionitis & $2(1.1)$ \\
\hline Peripartum maternal fever & $9(5.0)$ \\
\hline
\end{tabular}

PROM: prolonged rupture of membranes; UTI: urinary tract infection; GBS: group B streptococcus.

$\mathrm{P}$ value $=0.049)$, higher rates of positive CRP at $48 \mathrm{~h}$, and a longer hospital stay $(10 \pm 3$ days vs. $4 \pm 2$ days, $\mathrm{P}$ value $=$ 0.000) (Table 4).

\section{Discussion}

Management of asymptomatic newborns with PROM is puzzling. The American Academy of Pediatrics proposed guidelines for their management that depend on several factors [16, 17]. Most importantly was clinical status; any critically ill newborn should be given empirical antibiotics. As for asymptomatic neonates, management depends on gestational age and other risk factors, mainly GBS, intrapartum antibiotic coverage, and laboratory tests results.

GBS is the leading causative agent of EOS worldwide [18]. In the USA, the Centers for Disease Control and Prevention (CDC) recommends a universal screening for GBS in pregnant women around the 35 th week of gestation [19], and studies in the UK reported a decline in GBS infection rates when following a screening protocol [20]. However, there is a peculiar lack of GBS screening efforts in Jordan despite significant carriage rate among pregnant women [21]. In our study, the GBS status of all the mothers $(100 \%)$ was unknown, which was a major additive risk factor for neonatal sepsis in our cohort.

Of the 176 newborns $\geq 34$ weeks' gestation who were included, $32(18 \%)$ had sepsis, and nine of them $(5 \%)$ had culture proven sepsis. This rate of sepsis is slightly higher than previously reported rates $[15,22]$. The higher rate in our cohort might be exaggerated due to our definition of sepsis where neonates with positive CRP at $48 \mathrm{~h}$ were considered septic. For the sake of this study, we did not consider any positive blood culture as contamination as all the organisms found in our blood cultures were previously reported as neonatal sepsis causative agents. Furthermore, since we are planning to draft a protocol for management of these infants, considering all abnormal results as causative agents will help reach a safer protocol. The lack of GBS intrapartum chemoprophylaxis is a major factor for higher rates of EOS. Other factors like intrapartum obstetric practice variation, such as not restricting manual examination frequency, although not investigated in this study,
Table 3. Clinical Characteristics and Outcomes of Neonates $\geq$ 34 Weeks' Gestational Age Admitted due to Prolonged Rupture of Membranes

\begin{tabular}{|ll|}
\hline Characteristic & Number (frequency) \\
\hline $\begin{array}{l}\text { Status at birth } \\
\text { Well }\end{array}$ & $131(74.4)$ \\
\hline \multicolumn{1}{|c|}{ Ill } & $4(2.3)$ \\
\hline Equivocal & $45(25.6)$ \\
WBC $>25,000$ & $7(4.0)$ \\
WBC $<5,000$ & $2(1.1)$ \\
Thrombocytopenia & $11(6.3)$ \\
CRP baseline positive & $11 / 56(19.6)$ \\
CRP positive at $48 \mathrm{~h}$ & $40 / 147(27.2)$ \\
Blood culture positive & $9(5.1)$ \\
Length of hospital stay (average \pm SD) & $5 \pm 3$ \\
Mortality & $1(0.57)$ \\
\hline
\end{tabular}

WBC: white blood cell; CRP: C-reactive protein.

might provide explanation for the higher rate of EOS.

When a neonate shows symptoms at birth, empirical antibiotics are indicated. The difficulty arises when these infants are asymptomatic. In this situation laboratory investigation should be used to guide decisions, mainly CBC and CRP values. The utility of $\mathrm{CBC}$ in predicting those with sepsis has been investigated and proven unreliable [23]. In our cohort none of the infants with sepsis has leukocytosis, and there was no difference between infants with sepsis and normal newborns regarding leukopenia ( $3 \%$ vs. $1.4 \%, \mathrm{P}$ value $=0.492)$. Using CRP is not very helpful at the onset of illness [24]. Serial CRP readings have more value [13] which helps in the follow-up of infants at risk who did not receive empirical antibiotics. In those who received antibiotic therapy, CRP is useful in monitoring treatment response $[25,26]$. In neonates who had CRP done at $6-12 \mathrm{~h}$ of age, there was no significant difference between those with sepsis and normal newborns $(28.6 \%$ vs. $21.2 \%, \mathrm{P}$ value $=0.656)$. However, at $48 \mathrm{~h}$ there was a significant difference between the two groups $(43.8 \%$ vs. $27.54 \%$, P value $=0.008)$. This significance is subject to selection bias since rising $\mathrm{CRP}$ at $48 \mathrm{~h}$ is part of the criteria to define neonates with sepsis, as this difference illustrates the importance of serial CRP measurements.

The other tool that can be utilized in decision making for asymptomatic newborns is the sepsis risk calculator. It is considered a helpful tool for prediction of sepsis in neonates with infection risk factors [14]; however it is not completely reliable in predicting sepsis in normal newborns and has been shown to miss neonates with true sepsis $[12,22]$. Applying sepsis calculator retrospectively has some limitations, relying on the medical charts, and assuming maternal temperature [22], but in this case where drafting a protocol is the aim, it is an acceptable method. The most important step when using the calculator tool is determining the general condition of the newborn. "Well" and "ill" conditions are simple to decide. The "equivocal" condition is tricky and might not be a univer- 
Table 4. Comparison of Neonatal Characteristics and Outcomes Between Normal Newborns and Those With Sepsis

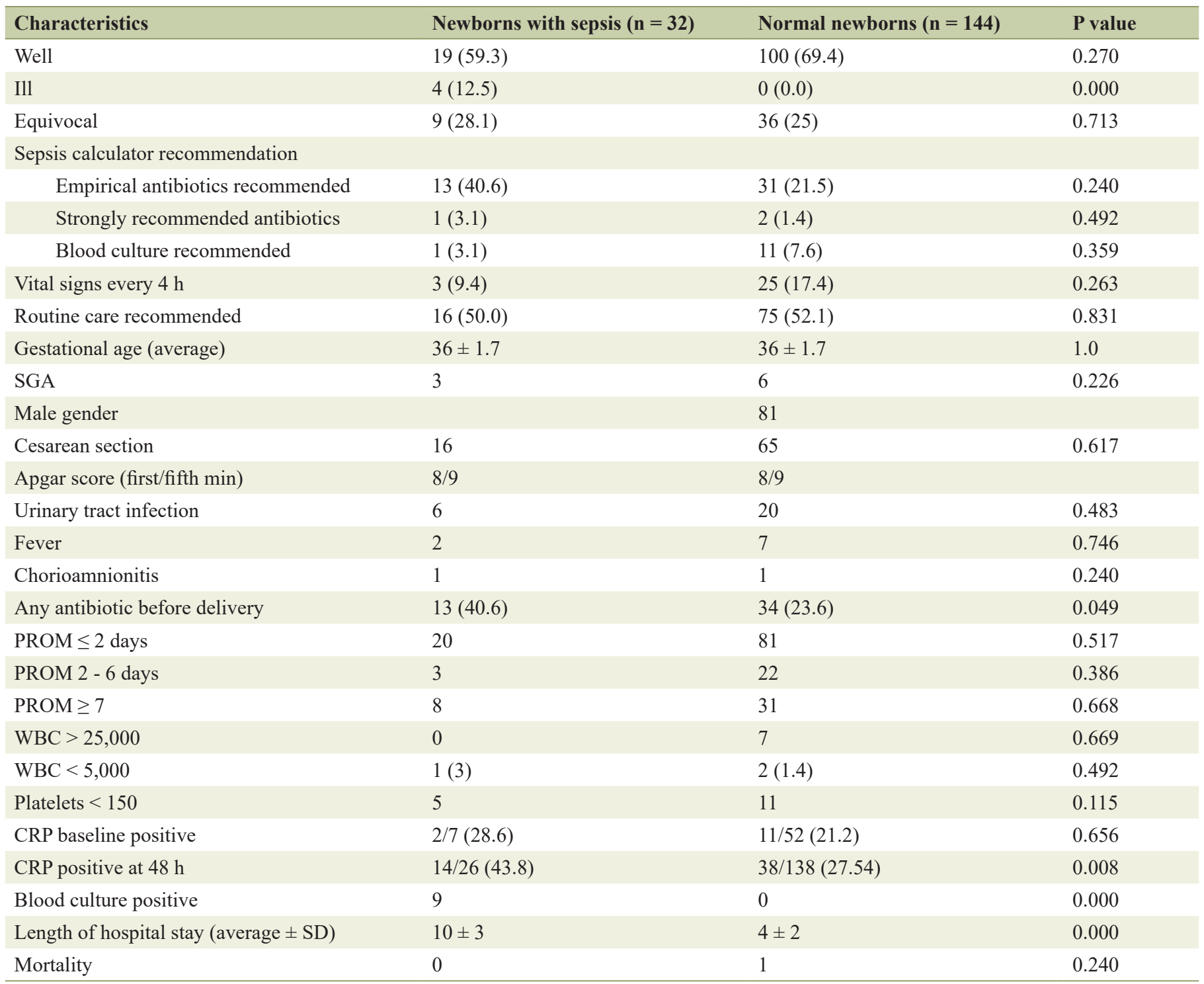

SGA: small for gestational age; PROM: prolonged rupture of membranes; WBC: white blood cell; CRP: C-reactive protein.

sally applied definition in all units. According to the calculator tool, the need for CPAP outside the delivery room automatically puts the newborn in the "ill appearing" category. On the other hand, the persistence of tachypnea and other respiratory distress symptoms beyond $4 \mathrm{~h}$ of age puts the newborn in the "equivocal" group. We believe this is confusing and contradicting. For a newborn who has prolonged respiratory transition, or respiratory distress signs persisting beyond the delivery room, our unit protocol indicates CPAP as treatment. The sepsis calculator tool placed the symptoms in one category and the therapy in a different one. Therefore, we suggest that since CPAP is the most widely used therapy for respiratory distress, neonates on CPAP therapy for mild to moderate respiratory distress who are hemodynamically stable and are improving should not be classified as "ill". In this study all our newborns with mild to moderate respiratory distress at birth were consid- ered "equivocal".

In this study, most infants $(67.6 \%)$ were classified "well" at birth. There was no significant difference in the "well" or the "equivocal" categories between the group with sepsis and the normal newborns $(59.3 \%$ vs. $69.4 \%$, $\mathrm{P}$ value $=0.27$ for the "well" group, and $28.1 \%$ vs. $25.0 \%$, P value $=0.713$ for the equivocal group). However when it comes to the "ill" category, none of the normal newborns were classified as "ill" $(12.5 \%$ vs. $0.0 \%, \mathrm{P}$ value $=0.000)$. This emphasizes the importance of the clinical status and stresses the importance of starting immediate antibiotics in newborns who are ill at birth.

Applying sepsis calculator in our cohort could have saved $77 \%$ of the neonates' unnecessary antibiotic treatment. However, the sepsis calculator recommendation could have missed $50 \%$ of our newborns with sepsis in whom the recommendation was routine care. Previous studies have shown similar 


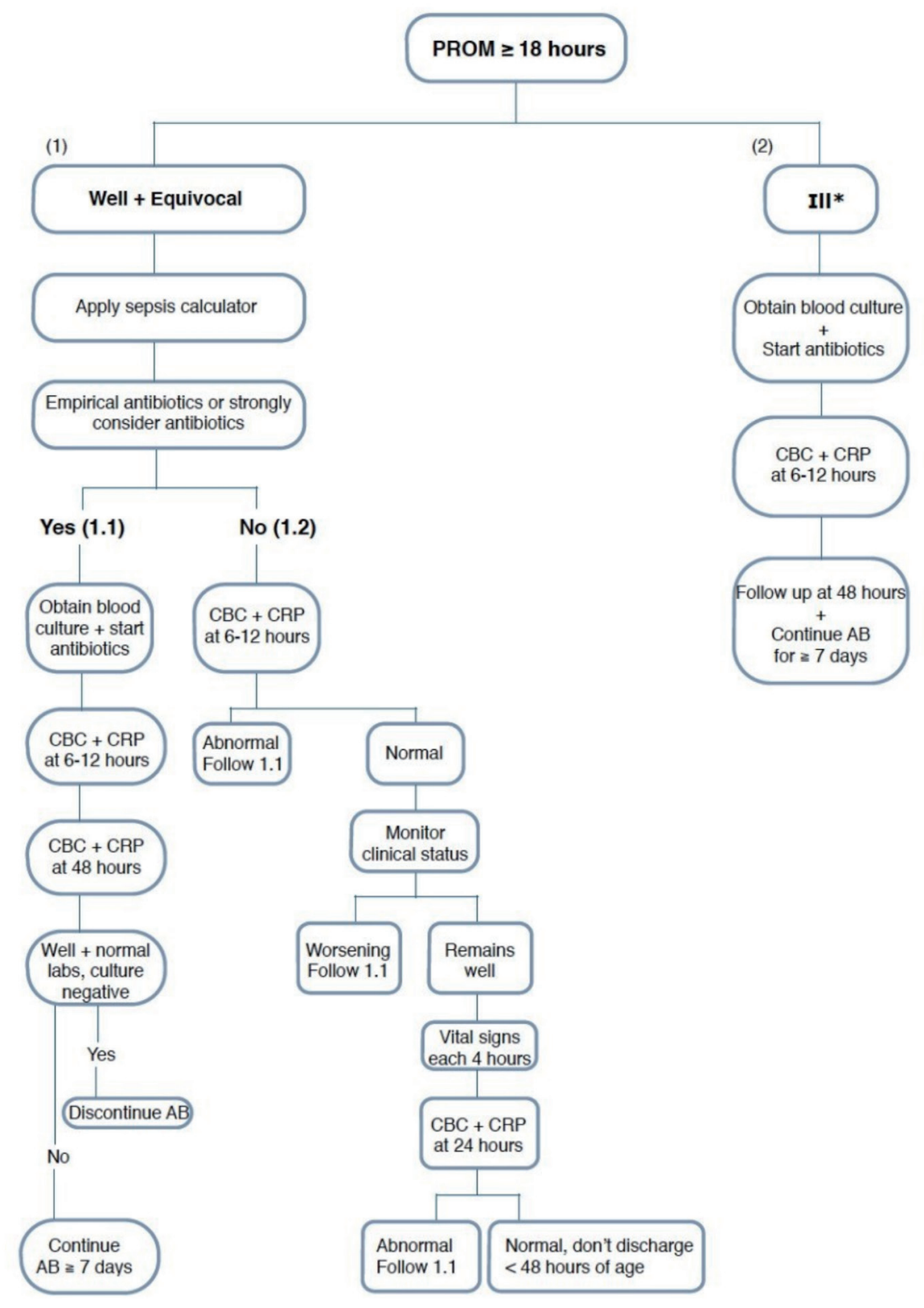

Figure 1. Suggested protocol of management of neonates born to mothers with prolonged rupture of membranes.

findings but to a lesser extent [15]. Sepsis calculator should be used with other diagnostic methods in order not to miss newborns with sepsis. Close monitoring of newborns with sepsis risk factors should be implemented regardless of their clinical status at birth or sepsis calculator recommendation. The follow-up strategy should combine clinical status and laboratory serial investigation.

There was no significant difference between the two groups regarding other maternal risk factors like the presence of urinary tract infection, maternal fever, or chorioamnionitis. However, the use of any antibiotics prior to delivery was significantly higher in the newborns with sepsis (40.6\% vs.
$23.6 \%$, P value $=0.049$ ). With the lack of GBS prophylaxis guidelines in our hospital, most of the included ladies received broad spectrum antibiotics for maternal illness shortly before birth, so they were considered non-protective when calculating sepsis risk factors [19]. The higher rate of antibiotics prior to delivery in those with sepsis might signify serious maternal illness that increased the risk of these newborns to sepsis.

It is clear that there is no single reliable tool in our setting that can be applied to predict which neonate is going to have sepsis. Applying multiple diagnostic methods, adopting strict follow-up strategy for those who do not receive antibiotics and keeping low threshold for antimicrobial treatment are 
very important especially at the beginning of changing current practice to insure a high safety profile of the newly adopted protocol.

Based on the current study and previously published guidelines [16, 17], our suggested protocol will depend on many factors for decision making regarding antimicrobial therapy in neonates with PROM that include clinical status, sepsis calculator recommendations, and laboratory investigations. Therapy will be started for ill newborns and when the sepsis calculator recommends starting antibiotics. For newborns who were not given antimicrobial therapy, at any time when their clinical status deteriorates or their laboratory investigations become abnormal, they will be switched immediately to the treatment group (Fig. 1).

This study among others is important to improve the practice of neonatology. Ensuring the transition in practice must be done safely as generating new recommended protocols is nation/setting specific. Our study has many limitations, with the main limitation being its retrospective nature. A prospective study is planned to investigate the usefulness of the suggested protocol.

Reliable early diagnostic tools for neonatal sepsis are lacking. Adopting a unit-specific protocol that utilizes multiple methods and applying strict follow-up for the clinical condition of these infants are the key factors to avoid missing neonates with true sepsis and decreasing the use of antibiotics in those without infection.

\section{Acknowledgments}

The authors thank all the NICU staff for their dedicated work.

\section{Funding}

No funding was received.

\section{Conflict of Interest}

The authors have no conflict of interest to declare.

\section{Informed Consent}

This is a retrospective chart review study. Informed consent is not applicable.

\section{Author Contributions}

MA contributed to the idea, design, analysis, and writing of the manuscript. AA and RE contributed to data gathering, analysis, drafting manuscript, and approving final manuscript. SA contributed to analysis, drafting manuscript, and approving final manuscript. EB contributed to data acquisition and approving final manuscript.

\section{References}

1. Mamede AC, Carvalho MJ, Abrantes AM, Laranjo M, Maia CJ, Botelho MF. Amniotic membrane: from structure and functions to clinical applications. Cell Tissue Res. 2012;349(2):447-458.

2. Canavan TP, Simhan HN, Caritis S. An evidence-based approach to the evaluation and treatment of premature rupture of membranes: Part I. Obstet Gynecol Surv. 2004;59(9):669-677.

3. Flenady V, King J. Antibiotics for prelabour rupture of membranes at or near term. Cochrane Database Syst Rev. 2002;3:CD001807.

4. Zeng LN, Zhang LL, Shi J, Gu LL, Grogan W, Gargano MM, Chen C. The primary microbial pathogens associated with premature rupture of the membranes in China: a systematic review. Taiwan J Obstet Gynecol. 2014;53(4):443-451.

5. Controlled study of influence on newborn of prolonged premature rupture of amniotic membranes and/ or infection in the mother. JAMA: J Am Med Assoc. 1963;184(4):181.

6. Boskabadi H, Maamouri G, Mafinejad S. Neonatal complications related with prolonged rupture of membranes. Macedonian Journal of Medical Sciences. 2011;4:93-98.

7. Alam MM, Saleem AF, Shaikh AS, Munir O, Qadir M. Neonatal sepsis following prolonged rupture of membranes in a tertiary care hospital in Karachi, Pakistan. J Infect Dev Ctries. 2014;8(1):67-73.

8. Simonsen KA, Anderson-Berry AL, Delair SF, Davies HD. Early-onset neonatal sepsis. Clin Microbiol Rev. 2014;27(1):21-47.

9. Drassinower D, Friedman AM, Obican SG, Levin H, Gyamfi-Bannerman C. Prolonged latency of preterm premature rupture of membranes and risk of neonatal sepsis. Am J Obstet Gynecol. 2016;214(6):743 e741-746.

10. Marlowe SE, Greenwald J, Anwar M, Hiatt M, Hegyi T. Prolonged rupture of membranes in the term newborn. Am J Perinatol. 1997;14(8):483-486.

11. Kiser C, Nawab U, McKenna K, Aghai ZH. Role of guidelines on length of therapy in chorioamnionitis and neonatal sepsis. Pediatrics. 2014;133(6):992-998.

12. Rajbhandari S, La Gamma EF. Early-Onset Sepsis Calculator-Risk of Delaying Treatment. JAMA Pediatr. 2017;171(10):1015.

13. Nuntnarumit P, Pinkaew O, Kitiwanwanich S. Predictive values of serial C-reactive protein in neonatal sepsis. J Med Assoc Thai. 2002;(85 Suppl 4):S1151-1158.

14. Carola D, Vasconcellos M, Sloane A, McElwee D, Edwards C, Greenspan J, Aghai ZH. Utility of early-onset sepsis risk calculator for neonates born to mothers with chorioamnionitis. J Pediatr. 2018;195:48-52 e41.

15. Polin RA, Committee on Fetus and Newborn. Management of neonates with suspected or proven early-onset bacterial sepsis. Pediatrics. 2012;129(5):1006-1015.

16. Benitz WE, Wynn JL, Polin RA. Reappraisal of guidelines for management of neonates with suspected earlyonset sepsis. J Pediatr. 2015;166(4):1070-1074. 
17. Schrag SJ, Zywicki S, Farley MM, Reingold AL, Harrison LH, Lefkowitz LB, Hadler JL, et al. Group B streptococcal disease in the era of intrapartum antibiotic prophylaxis. N Engl J Med. 2000;342(1):15-20.

18. Verani JR, McGee L, Schrag SJ, Division of Bacterial Diseases, National Center for Immunization and Respiratory Diseases, Centers for Disease Control and Prevention (CDC). Prevention of perinatal group B streptococcal disease-revised guidelines from CDC, 2010. MMWR Recomm Rep. 2010;59(RR-10):1-36.

19. Gopal Rao G, Nartey G, McAree T, O'Reilly A, Hiles S, Lee T, Wallace S, et al. Outcome of a screening programme for the prevention of neonatal invasive early-onset group B Streptococcus infection in a UK maternity unit: an observational study. BMJ Open. 2017;7(4):e014634.

20. Clouse K, Shehabi A, Suleimat AM, et al. High prevalence of Group B Streptococcus colonization among pregnant women in Amman, Jordan. American Journal of Obstetrics and Gynecology. 2017;217(6):739.

21. Kerste M, Corver J, Sonnevelt MC, van Brakel M, van der Linden PD, BA MB-L, Plotz FB. Application of sepsis calculator in newborns with suspected infection. J Ma- tern Fetal Neonatal Med. 2016;29(23):3860-3865.

22. Jackson GL, Engle WD, Sendelbach DM, Vedro DA, Josey S, Vinson J, Bryant C, et al. Are complete blood cell counts useful in the evaluation of asymptomatic neonates exposed to suspected chorioamnionitis? Pediatrics. 2004;113(5):1173-1180.

23. Hedegaard SS, Wisborg K, Hvas AM. Diagnostic utility of biomarkers for neonatal sepsis - a systematic review. Infect Dis (Lond). 2015;47(3):117-124.

24. Patil S, Dutta S, Attri SV, Ray P, Kumar P. Serial C reactive protein values predict sensitivity of organisms to empirical antibiotics in neonates: a nested case-control study. Arch Dis Child Fetal Neonatal Ed. 2016;101(6):F557F560.

25. Hofer N, Zacharias E, Muller W, Resch B. An update on the use of C-reactive protein in early-onset neonatal sepsis: current insights and new tasks. Neonatology. 2012;102(1):25-36.

26. Shakib J, Buchi K, Smith E, Young PC. Management of newborns born to mothers with chorioamnionitis: is it time for a kinder, gentler approach? Acad Pediatr. 2015;15(3):340-344. 